\title{
Diffusion-limited deposition of dipolar particles
}

\author{
F. de los Santos, ${ }^{1}$ J. M. Tavares,${ }^{2,3}$ M. Tasinkevych, ${ }^{4}$ and M. M. Telo da Gama ${ }^{2,5}$ \\ ${ }^{1}$ Departamento de Electromagnetismo y Física de la Materia, \\ Universidad de Granada, Fuentenueva, 18071 Granada, Spain \\ ${ }^{2}$ Centro de Física Teórica e Computacional da Universidade de Lisboa \\ Avenida Professor Gama Pinto 2, P-1649-003 Lisbon, Portugal \\ ${ }^{3}$ Instituto Superior de Engenharia de Lisboa \\ Rua Conselheiro Emídio Navarro, 1, P-1949-014 Lisbon, Portugal \\ ${ }^{4}$ Max Planck Institute for Metal Research, Heisenbergstrasse 3, 70569 Stuttgart, Germany \\ ${ }^{5}$ Departamento de Física, Faculdade de Ciências da Universidade de Lisboa, \\ R. Ernesto Vasconcelos, Lisbon, Portugal
}

(Dated: today)

\begin{abstract}
Deposits of dipolar particles are investigated by means of extensive Monte Carlo simulations. We found that the effect of the interactions is described by an initial, non-universal, scaling regime characterized by orientationally ordered deposits. In the dipolar regime, the order and geometry of the clusters depend on the strength of the interactions and the magnetic properties are tunable by controlling the growth conditions. At later stages, the growth is dominated by thermal effects and the diffusion-limited universal regime obtains, at finite temperatures. At low temperatures the crossover size increases exponentially as $T$ decreases and at $T=0$ only the dipolar regime is observed.
\end{abstract}

PACS numbers: $61.43 . \mathrm{Hv}, 64.60 . \mathrm{Cn}, 75.30 .-\mathrm{m}$

The growth of deposits by irreversible aggregation of particles is of great technological importance as well as of theoretical interest. A variety of mechanisms are involved in the growth processes but at late times scaling laws depending only on a few parameters have been observed. A general assumption, that describes the patterns found in many experiments, is that the deposition process is dominated by thermal diffusion. A simple model for this type of growth is diffusion-limited deposition (DLD) [1], characterized by the formation of branched, fractal structures similar to those found in electrodeposition, dielectric breakdown, etc (see 2] and references therein). Under certain circumstances, however, interparticle interactions, favoring ordered structures that compete with the randomness of the diffusion process, are required to describe the observed growth patterns. A case in point is the diffusion-limited deposition of magnetic particles subject to dipolar interactions.

Dipolar interactions are essential in determining the rich variety of structures exhibited by magnetic materials [3] , and their interplay with thermal diffusion may lead to novel magnetic properties. On the theoretical side, dipolar interactions provide a simple model to study the effects of anisotropic, long-ranged interactions on far from equilibrium aggregation processes. The central question concerns the change in the fractal dimension of the aggregates, $D$, as the dipolar interactions are switched on. Meakin et al. [4] considered the effect of isotropic long-ranged, $1 / r^{\epsilon}$, interactions in reactionlimited cluster-cluster aggregation (CCA) models. They found that $D$ is unchanged for short-ranged interactions, i.e. for $\epsilon \geq 2 D_{o}$ where $D_{o}$ is the fractal dimension of the non-interacting aggregates, while for longer-ranged in- teractions $D$ may change substantially. Accordingly, numerical results for diffusion-limitted aggregation (DLA), performed for particles with Ising spins (short-range interactions), revealed no changes in the fractal dimension of the aggregates with increasing exchange interactions [5]. Finally, results for DLA [6] of dipolar particles indicate that $D$ decreases as the strength of the dipolar interactions increases, in line with the results for CCA of dipolar particles [7] and with experimental results for the aggregation of magnetic microspheres [8], but in disagreement with preliminary results of ours 9 ].

In this article we report results of extensive Monte Carlo simulations that provide a general framework where the apparently contradictory results described above may be understood. We show that the initial stage of two dimensional dipolar DLD growth is indeed described by a new nonuniversal scaling regime, characterized by clusters (trees) whose shape and fractal dimension are temperature-dependent. For large enough systems, however, the dipolar regime crosses-over to the diffusion driven universal regime, where the effect of the dipolar interactions is dominated by thermal effects. It is also shown that the dipolar regime corresponds to orientationally ordered deposits and that the onset of the universal regime coincides with the disappearance of the orientational order. At $T=0$ only the dipolar regime is observed.

In the new dipolar regime, the orientational order as well as the shape and fractal dimension of the clusters depend on the strength of the interactions. Thus, the magnetic properties of dipolar deposits may be tuned by controlling the growth conditions, such as temperature. Finally, we found that the fractal dimension of the entire 

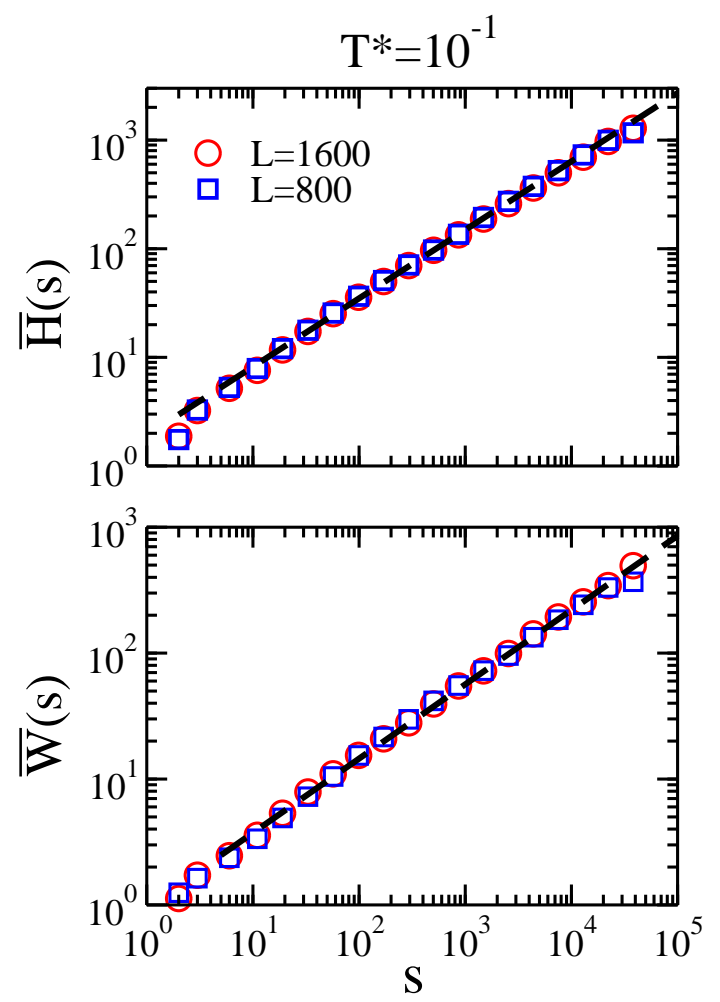

FIG. 1: (Color online) Average height $\bar{H}(s)$ and width $\bar{W}(s)$ as a function of the size of the tree, $s$, at $T^{*}=10^{-1}$ and various system sizes. The lines are power laws (2) with the exponents listed in table I. Dashed line: universal regime.

deposit is always given by the universal (diffusion driven) value implying that in the dipolar regime, the trees have a fractal dimension $D_{t}$ that differs from that of the entire deposit.

We consider a two-dimensional box of side $L$ and height $H$, on a square grid of side $a=1$. Periodic boundary conditions are applied parallel to the substrate, of size $L$, and particles are dropped from a finite height above it. The particles carry a dipole moment of strength $\mu$ and interact through the pair potential

$$
\phi_{1,2}=-\frac{\mu^{2}}{r_{12}^{3}}\left[3\left(\hat{\boldsymbol{\mu}}_{1} \cdot \hat{\mathbf{r}}_{12}\right)\left(\hat{\boldsymbol{\mu}}_{2} \cdot \hat{\mathbf{r}}_{12}\right)-\hat{\boldsymbol{\mu}}_{1} \cdot \hat{\boldsymbol{\mu}}_{2}\right],
$$

where $r_{12}$ is the distance between particles 1 and $2, \hat{\mathbf{r}}_{12}$ is the two-dimensional unit vector along the interparticle axis, and $\hat{\boldsymbol{\mu}}_{1}$ and $\hat{\boldsymbol{\mu}}_{2}$ are the three-dimensional unit vectors in the direction of the dipole moments of particles 1 and 2 respectively. A particle is released at a height $H_{\text {in }}$ with a dipole moment oriented at random. The particle undergoes a random walk through a series of jumps to nearest-neighbor sites, while interacting with the particles in the deposit. At each step a new position and a new random 3-dimensional dipole orientation are accepted according to a simple Metropolis rule based on the difference between the dipolar energies of the two configurations and defining the effective temper-
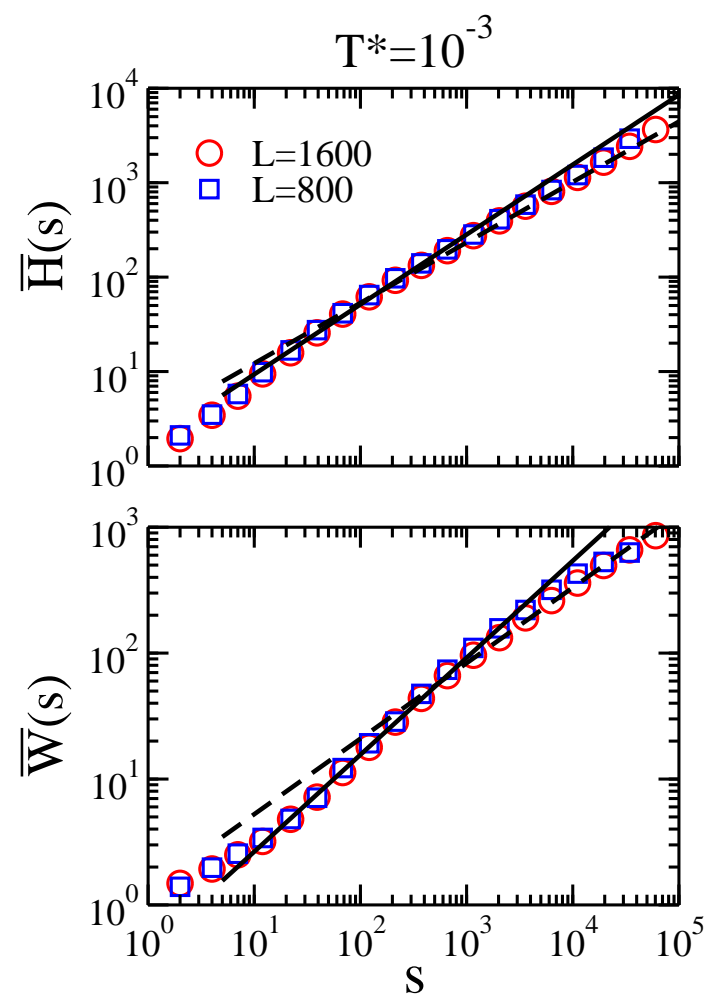

FIG. 2: (Color online) Average height $\bar{H}(s)$ and width $\bar{W}(s)$ as a function of the size of the tree, $s$, at $T^{*}=10^{-3}$ and various system sizes. The lines are power laws (2) with the exponents listed in table I. Full line: dipolar regime. Dashed line: universal regime.

ature $T^{*}=k_{B} T a^{3} / \mu^{2}$. The long range of the dipolar interactions is taken into account by an Ewald summation for the slab geometry of the system $[9$. In the limit $T^{*} \rightarrow \infty$ all displacements are accepted and the model reduces to DLD. A particle will eventually $(i)$ contact the deposit or the substrate sticking to it irreversibly as its dipole relaxes along the local field [10]; or (ii) reach a height greater than $H_{\text {out }}$, when it is removed and a new particle released. See [9, 11] for the details of the simulation.

Simulations were carried out at 4 temperatures, $T^{*}=10^{-1}, 10^{-2}, 10^{-3}, 10^{-4}$, and 4 system-sizes, $L=$ $200,400,800,1600$ with $20,30,50$, and 100 thousand particles per deposit, respectively. The deposits are similar to those of DLD: they consist of many small trees competing to grow [9]. As the number of particles in the deposit increases, fewer and fewer trees keep on growing due to shadowing until only a single tree survives.

We start by investigating the dependence of the height, $H$, and the width, $W$, of a tree with its size $s$ (number of particles), as well as the distribution of trees $n_{s}$, i.e. the average number of trees of a given size. In DLD the trees scale as 2, 12, 13,

$$
H \sim s^{\nu_{\|}}, \quad W \sim s^{\nu \perp}, \quad n_{s} \sim s^{-\tau} .
$$



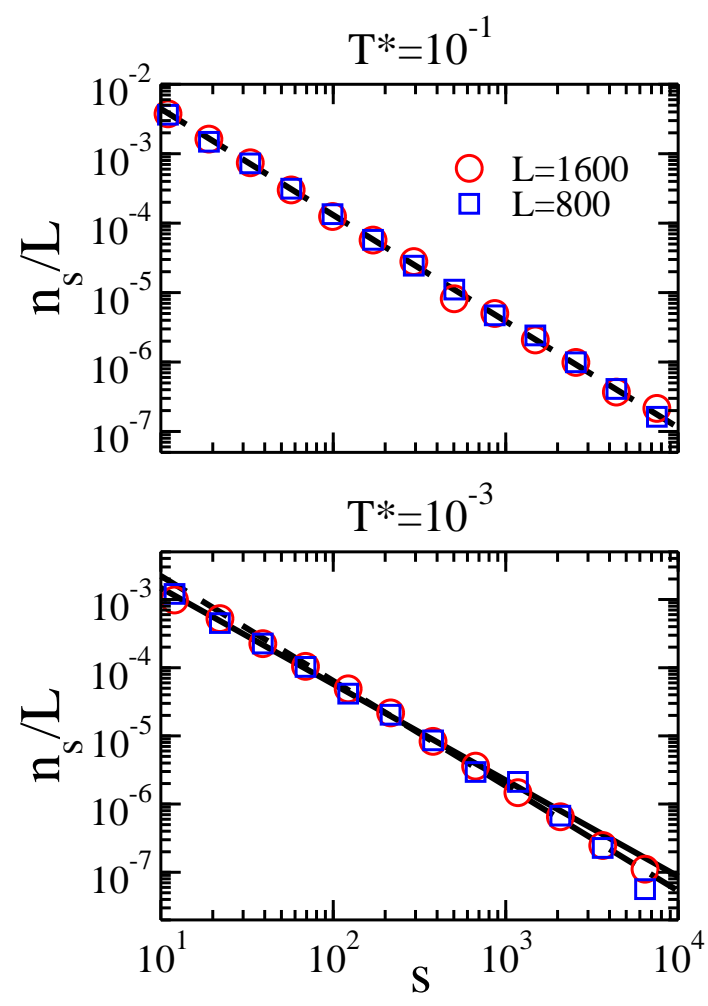

FIG. 3: (Color online) Average number of trees of size $s$ divided by $L\left(\bar{n}_{s} / L\right)$ as a function of $s$, at various temperatures and system sizes. Lines as in figure 2 .

for sufficiently large values of $s$. Owing to the finite size of the simulation box, however, only a single tree survives when the number of deposited particles is large enough and, thus at large $s$ the width of the tree saturates $(W \approx$ $L)$, then the height grows linearly with its size, $(H \propto s)$, and $n_{s}$ exhibits a discontinuity.

The difference between $\nu_{\|}$and $\nu_{\perp}$ measures the anisotropy of the trees. If $\nu_{\|}=\nu_{\perp}$ the trees are isotropic and their fractal dimension is $D_{t}=1 / \nu_{\|}$, whereas if anisotropy is present, $D_{t}$ becomes [2]

$$
D_{t}=1+\left(1-\nu_{\|}\right) / \nu_{\perp} .
$$

We note that the assumption that the deposit has the same fractal dimension as the trees, $D=D_{t}$, which holds in DLD, is not warranted in general. $D$ was estimated through the average particle density at height $h, \rho(h)$, which was found to scale as in DLD: at early times the deposit builds up until it reaches a height $h_{i}$. Then, we found a scaling regime where the density decreases as a power law of the height, $\rho(h) \sim h^{-\alpha}$, with $D=2-\alpha[2]$. The density saturates when the lateral correlation length $\xi_{\|}$reaches the size of the system, at $h_{s} \sim L^{\gamma}$. Simple arguments show [2] that the exponents $\nu_{\|}, \tau$ and $\alpha$ are related through

$$
\alpha=1-(2-\tau) / \nu_{\|}
$$

We have verified (2) by calculating the average maximal height and width of the largest tree, $\bar{H}(s)=\langle h(s)\rangle$, $\bar{W}(s)=\langle w(s)\rangle$, as a function of the tree size, $s$, and $\bar{n}_{s}=\left\langle n_{s}\right\rangle . h(s)$ and $w(s)$ are the maximal height and width of the largest tree of each deposit, and $\langle\ldots\rangle$ is an average over all the deposits.

Figures 1 and 2 show the results for $\bar{H}(s)$ and $\bar{W}(s)$ obtained at $T^{*}=10^{-1}$ and $T^{*}=10^{-3}$ for different box sizes. The points are histograms on a logarithmic scale. As expected, the results at $T^{*}=10^{-1}$ correspond to those of DLD. Scaling was found for a wide range of $s$ $(s>10)$, after a crossover from the early stage regime $(s<10)$. The crossover to the linear regime (only-onetree growth) was observed only for the smallest system, $L=200$, for $s>5000$ (not shown). $n u_{\|}$and $\nu_{\perp}$ calculated for each $\mathrm{L}$ were found independent of system size; a representative value was obtained by averaging over all $L$ with the uncertainty estimated as the largest deviation from the mean. Power law regressions for different ranges of $s$, yield $\nu_{\|}=0.64(1)$. The crossover to saturation of the width was observed for sizes up to $L=800$. Fitting only points that exhibit clear data collapse yields $\nu_{\perp}=0.60(2)$. These exponents agree with previous results for DLD [12], and show that at high temperatures, the universal behavior of the geometrical properties of the trees is unaffected by the dipolar interactions.

At $T^{*}=10^{-3}$, however, the existence of two scaling regimes is apparent, in particular for the largest system, $L=1600$, where the width saturation and the linear regimes are not observed. For $s$ less than the crossover size $s^{*} \approx 500$ we found $\nu_{\|}=0.74(1)$ and $\nu_{\perp}=0.78(2)$. These exponents differ from those of DLD and characterize a new growth regime that we call the dipolar regime. For $s>s^{*}$, we obtained, $\nu_{\|}=0.64(1)$ and $\nu_{\perp}=0.60(2)$, in line with the results for DLD. Similar behavior was observed at the other two temperatures, that is, a dipolar regime with temperature-dependent exponents followed by a second scaling regime with DLD exponents.

In table I we list the exponents of the dipolar and universal regimes, obtained at various temperatures. These results indicate that, if (2) is assumed, the effect of the dipolar interactions may be described by the appearance of a dipolar regime characterized by non-universal exponents $\nu_{\|}$and $\nu_{\perp}$ that increase with decreasing temperature. The crossover to the universal regime occurs at tree sizes that increase as the temperature decreases. As a consequence, the universal regime is difficult to observe at very low temperatures. However, at finite $T^{*}$, the universal regime may be reached if large enough deposits are grown. Indeed, we found no dependence of the crossover between the dipolar and the universal regimes on $L$, and thus at any temperature there is an $L$ above which this crossover may be observed. This implies that the geometrical properties of the trees may be tuned by controlling the dipolar interactions and the system size. In particular, it is possible to deposit trees with a given anisotropy 
by growing trees (at a fixed temperature) with a fractal dimension that is determined by the tree size. Alternatively, at fixed tree size, one may control the anisotropy by changing the temperature.

In figure 3 , we plot the results for the tree distribution at $T^{*}=10^{-1}$ and $T^{*}=10^{-3}$. At $T^{*}=10^{-1}$, the results are those of DLD. Scaling behavior was observed for a range of $s$ between 10 and the maximum tree size (which depends on $L$ ), with an exponent $\tau=1.54(2)$ in line with results for DLD 12 . At $T^{*}=10^{-3}$, assuming the crossover to occur at $s^{*} \approx 500$, we estimated $\tau$ in the ranges $10<s<s^{*}$ (dipolar regime) and $s^{*}<s<5000$ (universal regime). We found $1.40(2)$ and 1.56(4), respectively, for both systems. Thus, as for the tree height and width, we found a dipolar regime for the tree distribution, characterized by a temperature-dependent exponent. Again, the crossover to the universal regime occurs for sizes that increase as the temperature decreases. This crossover was not observed at $T^{*}=10^{-4}$ : due to the limited number of deposits and decreasing density, the number of trees larger than $s \approx 500-1000$ was too small to be analyzed. Thus, at this temperature only the dipolar regime was observed, with $\tau=1.36(3))$.

We have used (3) and (4) to verify the consistency of the exponents and to estimate $D_{t}$. In addition, we have checked the validity of (4) in the dipolar regime and compared $D_{t}$ in both regimes. $\alpha$ was estimated in both regimes by power law regressions of $\rho(h)$ in the regions suggested by the plots of $\bar{H}_{l}$ (using data from $L=1600$ ). We found weak crossovers at the temperatures $T^{*}=10^{-2}$ and $10^{-3}$, similarly to what was observed for $\bar{n}_{s}$. The results listed in Table I indicate a remarkable consistency between the values of $\tau$ obtained from simulation and using (4). It is also clear that the fractal dimension of the trees in the dipolar regime decreases with decreasing temperature, in line with previous studies of DLA dipolar aggregates [6, 7].

The connection between the orientational order of the dipoles and the geometrical properties of the deposits was investigated by comparing the mean-square magnetization density in the $x$ - and $y$ - directions at height $h,\left\langle m_{x, y}^{2}(h)\right\rangle$, with the mean particle density at the same height, $\rho(h)$. These results are shown in figure 4 . It is apparent that the density saturates at the same height as the mean-square magnetizations, and that the onset of the scaling behavior of $\rho$ coincides with that of $\left\langle m_{y}^{2}\right\rangle$. Notice also the height $h^{\dagger} \approx 300$, where $\left\langle m_{x}^{2}\right\rangle=\left\langle m_{y}^{2}\right\rangle$ and the orientational order vanishes. That $h^{\dagger}$ signals the disappearance of orientational order is seen most clearly in the inset of figure 3 . The latter shows that the diagonal elements, $Q_{x x}(h)$ and $Q_{y y}(h)$, of the ordering matrix $\mathbf{Q}$ become identical at $h=h^{\dagger}$, beyond which $Q_{x x}=Q_{y y}=1 / 4\left[11\right.$. We also found that $h^{\dagger}$ does not depend on $L$, but increases with decreasing $T^{*}$. At low $T^{*}$, the increase is exponential, $h^{\dagger} \simeq \exp \left(1 / T^{*}\right)$. This

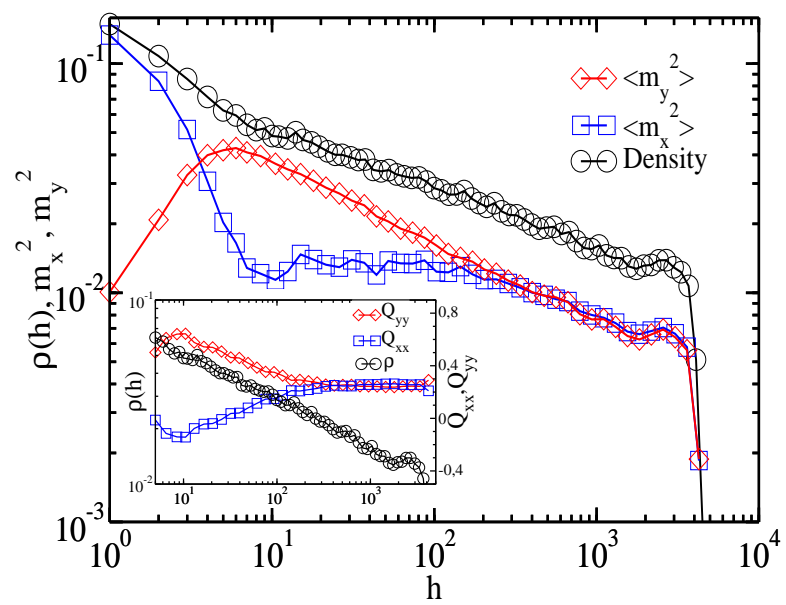

FIG. 4: (Color online) Average particle density and meansquare magnetization densities at height $h$. Inset: diagonal elements, $Q_{x x}$ and $Q_{y y}$, of the ordering matrix $\mathbf{Q}$.

functional dependence was obtained from a direct estimation of the heights at which the orientational order of the deposits vanishes. Depending on the temperature and system size, $h^{\dagger}$ is larger or smaller than $h_{s}$ and this is related to the existence of the dipolar and universal regimes: when $h^{\dagger}<h_{s}$ both the universal and the dipolar regimes occur, but only the latter is found when $h^{\dagger}>h_{s}$. We recalculated the scaling exponents assuming that the crossover between the dipolar and universal regimes occurs at $h=h^{\dagger}$ (equivalently, at $s^{\dagger}$ estimated using $\bar{H}(s)$ ) and found that they are identical with those of table I. Therefore, the dipolar regime is characterized by deposits with orientational order. As a consequence, and by contrast to what happens in DLD, the fractal dimension of the deposits depends both on the fractal dimension of the trees, $D_{t}$, and on the distribution of the trees, $\tau$. In the light of this, the decreasing $D_{t}\left(T^{*}\right)$ reported in [7, 8], is to be expected, since it was determined from the radius of gyration versus the (small) number of particles of each cluster. Furthermore, the results of [6], obtained for a single DLA cluster, may be interpreted as the crossover from the temperature-dependent fractal dimension at short length scales, to $D \simeq 1.7$ at long length scales [6].

We have also estimated the average interaction of a dipolar particle with similar particles in ordered and randomly oriented deposits numerically, and confirmed that for ordered deposits the interaction decays more slowly than $2 D_{o}\left(\equiv 2 \times D_{t}(T=\infty) \approx 3.28\right)$ while for random ones it decays faster, in line with the results of [4] for isotropic systems [14]. Finally, we have checked that the global fractal dimension of the deposits is unaffected (or very weakly affected) by the dipolar interactions. This was found by analyzing the decay of the mean density with $h$, the scaling of $h_{s}$ and of the density at saturation with $L$, the two-point density-density correlation function, the initial divergence of the interface width, and 
TABLE I: Characteristic exponents of the dipolar DLD model. $\nu_{\|}, \nu_{\perp}$ and $\tau$ obtained from the simulation; other exponents obtained using the equations indicated in brackets.

\begin{tabular}{|c|c|c|c|c|c|c|c|c|}
\hline$T^{*}$ & regime & $\nu_{\|}$ & $\nu_{\perp}$ & $\tau$ & $\alpha$ & $\tau$ (4) & $D_{t}$ (3) & $D_{t} \equiv 1 / \nu_{\|}$ \\
\hline$\infty(\mathrm{DLD})$ & universal & $0.630(2)$ & $0.580(4)$ & $1.56(2)$ & $0.288(2)$ & $1.551(3)$ & $1.64(1)$ & $1.59(1)$ \\
\hline $10^{-1}$ & universal & $0.64(1)$ & $0.60(1)$ & $1.54(2)$ & $0.29(1)$ & $1.55(1)$ & $1.60(3)$ & $1.56(2)$ \\
\hline $10^{-2}$ & dipolar & $0.70(3)$ & $0.69(3)$ & $1.46(3)$ & $0.27(1)$ & $1.49(3)$ & $1.43(8)$ & $1.43(6)$ \\
\hline $10^{-2}$ & universal & $0.63(2)$ & $0.60(4)$ & $1.53(4)$ & $0.30(1)$ & $1.56(2)$ & $1.63(7)$ & $1.59(5)$ \\
\hline $10^{-3}$ & dipolar & $0.75(2)$ & $0.77(2)$ & $1.40(2)$ & $0.24(1)$ & $1.44(2)$ & $1.32(5)$ & $1.33(4)$ \\
\hline $10^{-3}$ & universal & $0.64(1)$ & $0.60(2)$ & $1.56(4)$ & $0.28(1)$ & $1.55(1)$ & $1.60(3)$ & $1.56(2)$ \\
\hline $10^{-4}$ & dipolar & $0.83(3)$ & $0.83(3)$ & $1.36(3)$ & $0.25(1)$ & $1.38(3)$ & $1.20(7)$ & $1.20(4)$ \\
\hline
\end{tabular}

the mean height of the upper surface. In every case no significant deviation from DLD was found 14].

Funding from the Portuguese FCT, contract $\mathrm{SFRH} / \mathrm{BPD} / 5654 / 2001$, and from the Spanish MCyT, project BMF2001-2841, is gratefully acknowledged.

[1] P. Meakin, Phys. Rev. A 27, 2616 (1983).

[2] P. Meakin, Fractals, scaling and growth far from equilibrium , Cambridge Universtiy Press, Cambridge (1998); T. Vicsek, Fractal growth phenomena, World Scientific (1989).

[3] K. De'Bell, A.B. MacIsaac, and J.P. Whitehead, Rev. Mod. Phys. 72, 225 (2000).

[4] P. Meakin and M. Muthukumar, J. Chem. Phys. 91, 3212 (1989).

[5] N. Vandewalle and M. Ausloos, Phys. Rev E 51, 597 (1995).

[6] R. Pastor-Satorras and J.M. Rubí, Phys. Rev. E 51, 5994
(1995); Prog. Colloid. Polym. Sci. 110, 29 (1998); J. Magn. Magn. Mater. 221, 124 (2000).

[7] P.M. Mors, R. Botet, and R. Jullien, J. Phys. A 20, L975 (1987).

[8] G. Helgesen, A.T. Skjeltorp, P.M. Mors, R. Botet, and R. Jullien, Phys. Rev. Lett. 61, 1736 (1988).

[9] F. de los Santos, M. Tasinkevych, J.M. Tavares, and P.I.C. Teixeira, J. Phys.: Condens. Matter 15, S1291 (2003).

[10] The relaxation of the dipole does not appear to be an essential ingredient of the algorithm, since the same $D$ has been found in [7] with and without dipole moment relaxation.

[11] J.M. Tavares, M. Tasinkevych, F. de los Santos, and M.M. Telo da Gama, Mol. Phys. 1011659 (2003).

[12] P. Meakin, Phys. Rev. B 30, 4207 (1984); P. Meakin, J. Kertész, and T. Vicsek, J. Phys. A 21, 1271 (1988); Z. Rácz and T. Vicsek, Phys. Rev. Lett. 51, 2382 (1983).

[13] P. Meakin and F. Family, Phys. Rev. A 34, 2558 (1986).

[14] J.M. Tavares et al., unpublished. 